\title{
LOCAL APPROACH FOR EVALUATING HEAT TRANSFER OF PRISMATIC ELEMENTS ON A FLAT SURFACE
}

\author{
Suprun T.T. \\ Institute of Engineering Thermophysics, National Academy of Sciences of Ukraine, Kyiv, suprun@secbiomass.com
}

\begin{abstract}
Heat-exchange prismatic elements located on a flat surface are typical for many technical applications. The purpose of the work is to develop local approach for evaluating heat transfer of working surfaces based on local control of thermophysical parameters in characteristic zones of the working environment, using the methods of heat and mass analogy and hot-wire anemometry. The local approach allows more accurately diagnosing the types of flow in the boundary layer of a streamlined element and thereby, the temperature state of individual prismatic elements and the entire arrangement as a whole is determined more accurately. For calculating the local heat transfer of each face of the prism separately and the average surface heat transfer depending on the geometric and operating parameters equations of similarity are proposed.
\end{abstract}

Keywords: prismatic heat exchange surface, local thermophysical parameters

\section{Introduction}

To improve the efficiency and reliability of the equipment it is necessary to constantly develop the methods of control and management of work processes. This is achieved on the basis of penetration into the complex mechanism of boundary layers development and targeted impact on their internal structure, which is typical for the general approach of the Institute of Engineering Thermophysics of the National Academy of Sciences of Ukraine to the management of transfer processes in the flow path of thermal power equipment for various purposes [1]. One of the most promising methods for studying transport processes occurring under complicated conditions is physical modeling $[2,3]$.

The object of this research is heat-exchange prismatic surfaces, which are typical for many technical applications. Increasing the intensity of transfer processes in heat exchange, chemical and technological equipment is often achieved using specially designed prismatic intensifiers located on flat surfaces. In some devices the presence of prisms on flat surfaces is due to the peculiarities of the technological process. In particular, prisms are typical elements of electronic equipment boards. Application of the method of local control of thermophysical parameters under conditions of action of turbulizing effects of various nature allows to optimize typical arrangement, to develop methods for calculating heat exchange of elements and units of electronic equipment, in turn, leads to a decrease in costs for product development, selection of the required power for a cooling system, improvement of equipment design, its reliability and resource. The experimental results presented below contribute to the solution of this urgent problem.

Until recently the most common approach for calculating the heat transfer of electronic equipment elements was another approach, which we called the channel one [4]. This approach is fundamentally different from the proposed local approach. With the channel approach the average heat transfer of prismatic elements is calculated using the similarity equation for a stabilized flow in channels using correction factors for inlet conditions, channel geometry and element arrangement. In this case the Reynolds number for all elements is determined by the average flow rate, thus it is assumed to be the same for any of the elements in a given channel, and diagnostics of the type of flow in the boundary layer that occurs on the element surface is made only on the basis of this Reynolds number, without dividing it into laminar, turbulent, pseudolaminar, quasi-turbulent, transient and separated flow regimes [5].

The limitations of this approach are obvious, since the information about the velocity and temperature fields is ignored, the type of flow is roughly diagnosed, and the influence of the configuration and size of elements and their location on the hydrodynamic structure of the flow is insufficiently taken into account. 


\section{Experimental technique}

To study the influence of turbulizing effects of various nature on the flow around prismatic elements located on a flat surface a special aerodynamic stand ADS-1 was created. The investigated flat surface with prismatic elements was installed in the working section of the ADS-1.The thermal efficiency of individual prismatic elements was tested for the arrangement shown in Fig. 1, which included four rows of prismatic elements. Turbulence in the first row was created by the input conditions and in the second, third and fourth rows - by the traces of the previous rows; flow separation occurred when flowing around the input edges of the elements. In Fig. 1 each prism is designated by a number, the first digit of which corresponds to the number of the row along the direction of the air flow, and the second to its ordinal number in the row. In this work we compared the average heat transfer between prism No. 23 located in position 1 (flow conditions 1) and prism No. 43 in position 2 (flow conditions 2). These conditions from the point of view of flow hydrodynamics were fundamentally different from each other, which will be described in detail below.

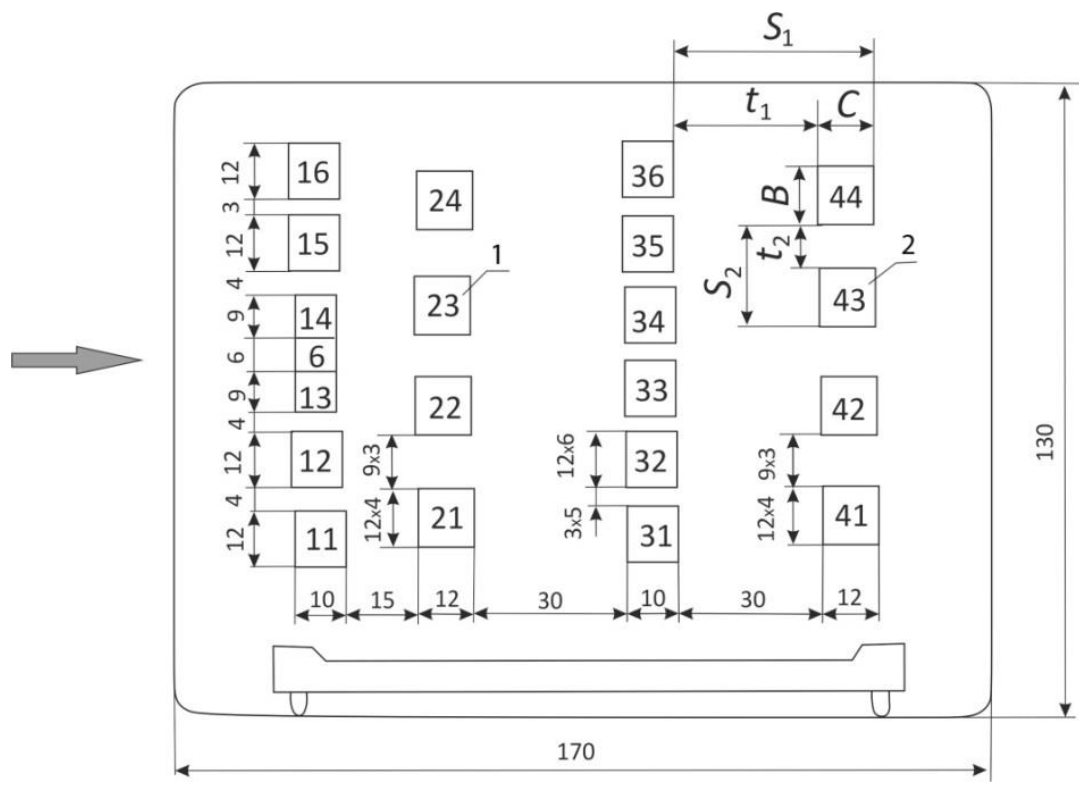

Fig.1. Flat surface with prismatic elements

For local control of thermophysical parameters in the channel of the ADS-1 working section, a hot-wire digital flow meter of the RPS-1 type developed by IET NAS of Ukraine was used, as well as hot-wire anemometer equipment DISA-55M. The aerodynamic features of the flow around the prismatic elements were determined based on the visualization of the flow using a soot-oil suspension applied to the prisms and aluminum powder, which was introduced into the air flow and whose particles adhered to the surface of the prism. Visualization was carried out both over individual prisms (for example, No. 23 for flow condition 1 and No. 43 for flow condition 2), and over the entire flat surface with installed prisms.

To study the heat transfer of prismatic elements, the method of heat and mass analogy was used, in particular, the method of sublimation of a substance in air. In this work, naphthalene was used as such a substance. The production of prismatic elements models was carried out using isobaric pressing technology. To determine the average mass loss from the surface of the model under study, an analytical balance of the VLR-200 type was used. The sample was weighed before and after the experiment. Local mass loss was determined by taking profilograms of the investigated surfaces of the models before and after the experiment on a special device.

\section{Results and discussion}

To determine the hydrodynamic structure of the flow in this study an assessment of the deviations of the local velocities over various prismatic elements $U_{i i}$ relative to their average flow rates in the working channel of the stand $\bar{U}$ was made (Table 1). As you can see from the table 1, the relative local velocities vary 
over a fairly wide range: from 0.47 to 1.20 . Therefore, the use of the average flow rate when carrying out the corresponding calculations in the general case can lead to significant differences between the calculated and actual values of the heat transfer coefficients of the prisms.

Table 1. Relative local flow velocities over different prismatic elements

\begin{tabular}{|c|c|c|c|c|c|c|c|c|c|c|c|}
\hline prism No. & 11 & 14 & 16 & 23 & 24 & 31 & 34 & 36 & 41 & 43 & 44 \\
\hline$U_{i i} / \bar{U}$ & 0.86 & 1.20 & 1.07 & 0.75 & 0.88 & 0.47 & 0.63 & 0.47 & 0.63 & 0.62 & 0.55 \\
\hline
\end{tabular}

As evidenced by the results of visualization, the separation zones located on the prisms and due to the features of their flow have different lengths: the maximum - in the final (fourth) row and the minimum - in the first. An increase in the length of the separation zones on the upper faces of the prisms along the length of the channel (flow conditions 1 and 2) indicates a general tendency for a decrease in the part of the kinetic energy of turbulence, which is perceived by the boundary layers developing on these faces, that is, it directly affects the process of their flow around. All these characteristic aerodynamic features of the flow around elements (flow conditions 1 and 2) have a significant effect both on the average heat transfer of these elements and on the local heat transfer coefficients. The determination of the average heat transfer was carried out for 11 prisms located in each of the four rows of this arrangement. The analysis of the results showed that the intensity of the average heat transfer for the prisms located in positions 1 (prism No. 23) and 2 (prism No. 43) is different, which is associated with the aerodynamic features of their flow.

The average heat transfer coefficient for prism No. 23 practically coincides with the values of the average heat transfer coefficients for the prisms located in the first three rows of the arrangement, and with an error of $\pm 7 \%$ are generalized by the dependence:

$$
\overline{N u}=C \operatorname{Re}^{0.6},
$$

where $C=0.536$.

An exponent of 0.6 at the Reynolds number indicates the existence of a pseudolaminar boundary layer (PLBL) on the surfaces of most prisms [6]. A specific feature of the PLBL is the intensification of heat transfer, which can be estimated by the ratio of the average heat transfer coefficients in it to their values in the laminar boundary layer, determined from the known dependence:

$$
\overline{N u}=0.59 \operatorname{Re}^{0.5}
$$

The intensification of heat transfer in the PLBL progresses with an increase in the Reynolds number. For 1-3 rows the intensification of the average heat transfer at Reynolds numbers of $10^{3}$ is $\sim 1.7-1.9$, and at $10^{4}-2.1-2.4$. Particular attention should be paid to the analysis of heat transfer for the prisms of the final (fourth) row (Fig. 2).

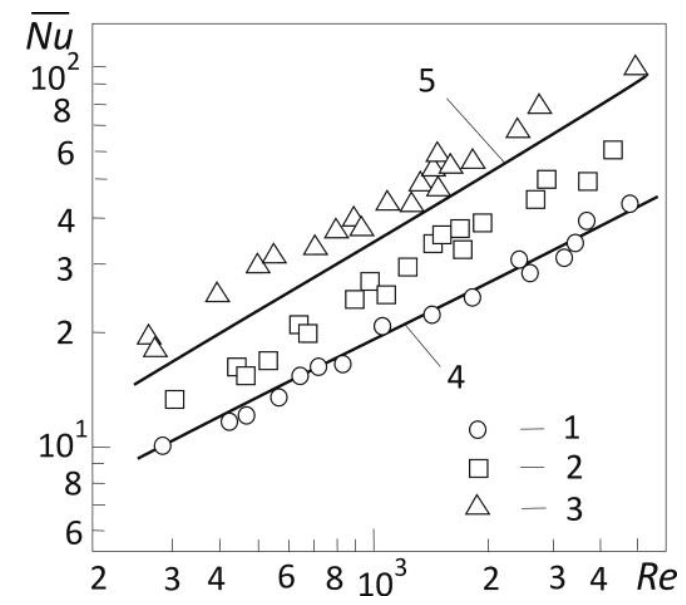

Fig.2. The average heat transfer of the last row of prisms located on a flat surface:

1 - prism No.41; 2 - prismNo.43; 3 - prismNo.44, 4 - eq. (2), 5 - eq. (1) 
As can be seen from Fig. 2, the elements of the fourth row are characterized by both laminar (prism No. 41) and pseudo-laminar flow regimes (prisms No. 43, 44) of various intensities, which are also generalized by dependence (1), where $C=0.34$ for prism No. 43 and $C=0.56$ for prism No. 44 . The average heat transfer of the last row of prisms is less intense than the heat transfer of the first three rows. So, for prism No. 43 the intensification of the average heat transfer at $\operatorname{Re} \approx 3 \cdot 10^{2}$ is $\sim 1.4$, and at $\operatorname{Re} \approx 3 \cdot 10^{3}-\sim 1.65$.

A comparative analysis of the heat transfer of an element under flow conditions 1 and 2 shows that during the constructive development of a flat surface with prismatic elements the most heat-stressed of them must be located under conditions of maximum heat removal. In this case such conditions turned out to be flow conditions 1 .This is a very important practical conclusion.

According to the local approach in equation (1) to calculate the average heat transfer it is necessary to use the local velocity over the prism. Taking into account the dependence $\left(U_{i i} / \overline{U_{n}}\right)^{n}$ connecting the local velocity over the prism $U_{i i}$ with the average flow rate in the channel $\overline{U_{n}}$, the similarity equation (1) takes the form:

$$
\overline{N u}=\overline{N u}_{c p}\left(U_{i i} / \overline{U_{n}}\right)^{n},
$$

where $\overline{N u}=C \operatorname{Re}^{n}, \overline{N u}_{c p}=C \operatorname{Re}_{c p}^{n}$, $\operatorname{Re}$ and $\operatorname{Re}_{c p}$ - Reynolds numbers, determined by local $\left(U_{i i}\right)$ and average $\left(\overline{U_{n}}\right)$ flow rates, respectively.

The determination of the local heat transfer coefficients was carried out on the basis of the distributions of local mass carryover on the faces of the prism model. The distributions of local heat transfer coefficients along the central axis of each face (at $U=5 \mathrm{~m} / \mathrm{s}, \operatorname{Re}=4200$ ) for prism No. 23 are shown in Fig. 3.

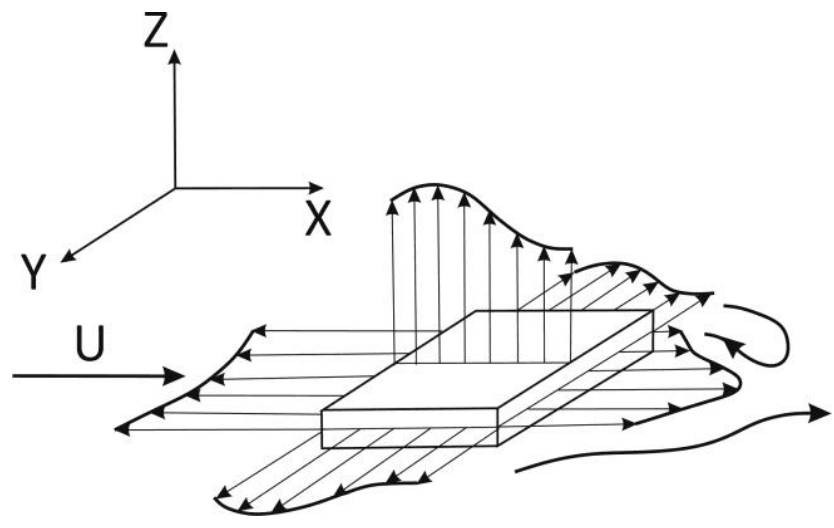

Fig.3. Distribution of local heat transfer coefficients along the central axes of the faces of prism No. 23

At the top and side faces a separated flow first occurs, which leads to the appearance in the distributions $\alpha=f(x)$ maxima located at distances $x_{r}$ from the leading edge of the element and corresponding to the point of reattachment of the flow. Behind this point, at $x>1.25 x_{r}$, the development of the PLBL begins.

The main results of the experimental study are presented in Table.2. They can be used for calculations of a single prismatic element mounted on a flat surface without a gap when flowing around a highly turbulized air flow. For the data presented in Table 2 in the investigated range of Reynolds numbers the heat balance for the entire element is observed with a maximum error of $+4.3 \%$ (at $\mathrm{Re}=500$ ). When carrying out these calculations, the average heat transfer coefficient of the entire element was calculated on the basis of the average heat transfer coefficients of each face determined from the table, taking into account its surface.

This approach allows us to single out the role of each face in the total heat transfer. So, for example, for a given element the contribution of the upper face is prevailing and amounts to $\sim 50 \%$ in the total heat removal (provided that the temperature difference is constant). At the same time, the highest are the heat transfer coefficients on the frontal face, which exceed by 1.6-2 times their value on the upper face, thereby compensating for the lower intensity of heat transfer on the back and side faces. 
Table 2. Recommendations for calculating the average heat transfer of a prismatic element and its faces in 1-3 rows gap-free installed on a flat surface in a stream

\begin{tabular}{|c|c|c|c|c|}
\hline \multicolumn{2}{|c|}{ General view of the similarity equation } & \multicolumn{3}{|c|}{$\overline{N u}=C \operatorname{Re}^{n}$} \\
\hline Average heat transfer & Defining size & Heat exchange surface & $C$ & 0.6 \\
\hline The element as a whole & $C$ & $\sum F$ & 0.536 & 0.6 \\
\hline The upper face & $C$ & $C \times B$ & 0.487 & 0.6 \\
\hline The side face & $C$ & $C \times h$ & $0.75-0.89$ & 0.5 \\
\hline The frontal face & $h$ & $B \times h$ & 0.116 & 0.667 \\
\hline The back face & $h$ & $B \times h$ & & \\
\hline
\end{tabular}

This leads to the fact that the total heat transfer coefficient practically coincides with the average heat transfer coefficient on the upper face. In this case, the contribution of the front face to the total heat transfer is only $19-22 \%$ (at $\operatorname{Re}=4000$ and 500 , respectively).

\section{Conclusions}

Complex experimental studies of heat and momentum transfer processes in difficult conditions of interaction of external (increased turbulence) and internal (separation) turbulizing effects typical for thermal power equipment have been carried out. A bank of new experimental data on the intensity of the processes occurring for a flat surface with prismatic elements has been obtained.

During the research a local approach was used, the advantage of which is the ability to fix the thermophysical parameters of the process in any characteristic zone of the working space. The meaning of the local approach is to determine the average surface heat transfer coefficient from the local velocity measured over each prismatic element. This makes it possible to assess the spatial temperature heterogeneity of the arrangement and, if necessary, by maneuvering the location of the elements, take measures to change the temperature in the desired direction.

Equations of similarity are proposed for calculating the local heat transfer of each face of the prism separately and the average surface heat transfer. Based on the recommendations received, the thermal state of a specific arrangement was assessed and measures were developed to improve it by purposefully rearranging the elements.

\section{REFERENCES}

1 Suprun T.T. Methods for managing transient processes on streamlined surfaces of power equipment. Thermophysics and Thermal Power Engineering, 2017, Vol.39, No.7, pp. 125. [in Ukrainian]

2 Suprun T. Physical modeling the unsteady flow with wakes. Eurasian phys. tech. j. 2017, Vol.14, No. 2(28), pp.113 - 119.

3 Suprun T.T. Simulation of turbulized flows typical for thermal power equipment. Thermophysics and Thermal Power Engineering, 2019, Vol. 41, No. 5, pp. 112. [in Ukrainian]

4 Gidalevich V.B., Mironenko Yu.P., Spokoyny Yu.E., Trofimov VE, Isaychenko V.V. Determination of local heat transfer coefficients of microcircuits with forced air cooling. Radio electronics issues.1983, Series TRTO, No.3, pp.3-9. [in Russian]

5 Dick E., Kubacki S. Transition Models for Turbo machinery Boundary Layer Flows: A Review. Int. J. Turbomach. Propuls. Power, 2017. Vol.2, No.2, pp.1-45. https://doi.org/10.3390/ijtpp2020004

6 Epik E.Ya., Suprun T.T. Impulse and heat transport processes at by pass transition. Eurasian phys. tech. $j$. 2007, Vol.4, No. 1(7), pp. 52 - 57. 